\title{
Silencing PDK1 limits hypoxia-induced pulmonary arterial hypertension in mice via the Akt/p70S6K signaling pathway
}

\author{
RUOMIN DI ${ }^{1}$, ZHONGZHOU YANG $^{2}$, PENG XU $^{1}$ and YINGJIA XU ${ }^{1}$ \\ ${ }^{1}$ Department of Cardiology, The Fifth People's Hospital of Shanghai, Fudan University, Shanghai 200240; \\ ${ }^{2}$ MOE Key Laboratory of Model Animal for Disease Study, Model Animal Research Center, \\ Nanjing University, Nanjing, Jiangsu 210061, P.R. China
}

Received September 18, 2017; Accepted June 15, 2018

DOI: $10.3892 / \mathrm{etm} .2019 .7627$

\begin{abstract}
The present study aimed to investigate the effect of phosphoinositide-dependent protein kinase-1 (PDK1) on hypoxia-induced pulmonary arterial hypertension $(\mathrm{PAH})$. A mouse model of hypoxia-induced PAH was generated using normal or $P D K 1$-knockout mice. Histological analysis and hemodynamic evaluations were performed to identify the progression of PAH. The expression and phosphorylation of PDK1/protein kinase B (Akt) signaling pathway associated proteins were detected by western blot analysis. Increased lung vessel thickness, right ventricular (RV) systolic pressure (RVSP), RV hypertrophy index (RVHI) values [the RV weight-to-left ventricular (LV) plus septum (S) weight ratio] and PDK1 expression were observed in the hypoxia-induced PAH model compared with the normal control. The phosphorylation of $\mathrm{Akt}^{\mathrm{T} 308}$, proline-rich Akt1 substrate 1 (PRAS40) and S6KT ${ }^{229}$ was also notably increased in the PAH model compared with the control. The changes of proteins were not observed in the hypoxia treated $P D K 1^{f o x /+}$ : Tie2-Cre mice. Similarly, the RVSP and RVHI values, and PDK1 expression were reduced in the hypoxia treated $P D K I^{f l o x /+}$ : Tie2-Cre mice to a level comparable with those in the control, suggesting that $P D K 1$ partial knockout significantly limited hypoxia-induced PAH. The results of the present study indicate that PDK1 is essential for hypoxia-induced PAH through the PDK1/Akt/S6K signaling cascades.
\end{abstract}

\section{Introduction}

Pulmonary arterial hypertension (PAH) is a progressive disease associated with the excessive proliferation of pulmonary vascular smooth muscle cells (pVSMCs) and pulmonary

Correspondence to: Dr Yingjia Xu, Department of Cardiology, The Fifth People's Hospital of Shanghai, Fudan University, 128 Ruili Road, Shanghai 200240, P.R. China

E-mail: anjiaozibbk3307@163.com

Key words: phosphoinositide-dependent protein kinase-1, pulmonary arterial hypertension, protein kinase $\mathrm{B}$, hypoxia vascular endothelial cells (pVECs), deposition of the extracellular matrix (ECM) and ECM remodeling, which results in a persistent increase in pulmonary arterial pressure with vascular remodeling and organ fibrosis.

It has been previously reported that the excessive proliferation of pVSMCs and pVECs is mediated by various signaling molecules, including protein kinase B (Akt) and transforming growth factor (TGF) $\beta$ (1). The Akt/mechanistic target of rapamycin (mTOR) signaling pathway is associated with the differentiation of myofibroblasts and extracellular remodeling, which are critical for organ fibrosis. mTOR signaling promotes cell proliferation and is essential for hypoxia-mediated pVSMC or pVEMC proliferation and angiogenesis (2). TGF $\beta$ may induce pulmonary fibrosis (3) and activate the phosphoinositide 3-kinase (PI3K)/Akt signaling pathway, which enhances fibroblast proliferation and the production of collagen (4). Lu et al (4) previously demonstrated that the inhibition of PI3K abrogated the effects of bleomycin, a fibrosis inducer that induces rapid lung fibrosis and activation of the PI3K/Akt signaling pathway, on fibroblast proliferation and collagen production.

Phosphoinositide-dependent protein kinase-1 (PDK1) serves a key role in the activation of the AGC subfamily of protein kinases, including Akt (5). A previous study revealed that PDK1-dependent AGC kinase activation is a requirement of Akt oncogenic activity (6). In addition, PDK1-dependent metabolic reprogramming dictates the metastatic potential of breast cancer cells (7). PDK1 is associated with the proliferation of cancer and basilar artery smooth muscle cells by Akt-mediated signals (8-10). Yu et al (11) recently revealed that tanshinone IIA induced the inactivation of PDK1 as well as the development of hypertension. Previous studies indicated that PDK1 is a hypoxia-responsive protein, which serves an important role in stress responses $(12,13)$. PDK1 deficiency results in heart failure and increased hypoxia sensitivity in cardiac muscle (14). However, to the best of our knowledge, the association between PDK1 and PAH has not yet been determined.

The present study aimed to investigate the effect of PDK1 on the development of PAH. A mouse model of hypoxia-induced PAH was generated using $P D K 1^{f f o x /+}$ : Tie2-Cre mice or control mice. The activation of the PDK1/Akt signaling pathway was detected by western blot; histological and hemodynamic 
analyses were also performed. The association between the PDK1-mediated Akt signaling pathway and hypoxia-induced PAH progression was evaluated.

\section{Materials and methods}

Animals and treatment. A total of 25 C57/BL6 male mice (age, 8 weeks; weight, 20-30 g) were purchased from Model Animal Research Center, Nanjing University (Nanjing, China) and maintained under stress-free conditions at $23 \pm 2^{\circ} \mathrm{C}$ with a humidity of 70\% and a 12-h light/dark cycle with food and water provided ad libitum. The PAH model was induced by hypoxia exposure in hypobaric chambers. The experimental animals (C57/BL6 mice, PDK1 $1^{\text {flox/+ }}$ mice and PDK $1^{\text {flox/+t }}$ : Tie2-Cre mice; $\mathrm{n}=15$, respectively) were exposed to chronic hypobaric hypoxia $\left(0.5 \mathrm{~atm}, 10 \% \mathrm{O}_{2}, 50 \%\right.$ humidity and $5 \% \mathrm{CO}_{2}$ at $\left.23 \pm 2^{\circ} \mathrm{C}\right)$ in chambers for 21 days and were let out three times per week as previously described (15). The control animals (C57/BL6 mice, PDK $1^{\text {flox/+ }}$ mice and PDK $1^{\text {flox } / \text { : }}$ : Tie2-Cre mice; $\mathrm{n}=10$, respectively) were exposed to normobaric chambers (room air) over the same 21-day period. Mice were anesthetized and received surgery following 21 days hypoxia exposure and pulmonary artery tissues were obtained following euthanasia by cervical dislocation and prepared for western blot or reverse transcription-quantitative polymerase chain reaction (RT-qPCR) analysis. The Institutional Animal Care and Use Committee of Shanghai Fifth People's Hospital approved the present study.

Generation of PDK1 partial knockout mice in endothelial

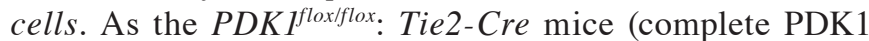
knockout in endothelial cells) succumbed during the embry-

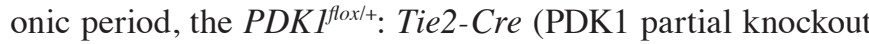
in endothelial cells) mice were generated by hybridization of PDK1 $1^{f o x / f o x}$ mice and Tie2-Cre mice (with Cre-expressing endothelial cells), which were provided by Model Animal Research Center, Nanjing University. The PDK1 genotyping was performed by PCR using DNA extracted from the tails of mice 7 days after birth. Different groups of mice, including C57BL6 mice, $P D K 1^{f f o x /+}$ mice $(\mathrm{n}=25)$ and $P D K 1^{f f o x /+}$ : Tie2-Cre mice $(\mathrm{n}=25)$ were selected for further study.

Semi-quantitative PCR. Genomic DNA was extracted from individual samples $(200 \mu \mathrm{l})$ using the DNeasy tissue kit (cat. no. 69506; Qiagen, Inc., Valencia, CA, USA) following the manufacturer's protocol and eluted in $100 \mu \mathrm{l}$. The percentage of agarose gel used in semi-quantitative PCR was $2 \%$ and samples were visualized using ethidium bromide. The primers of PDK1 were as follows: Forward, 5'CTCTACCTCCACCATGCCAAG T3' and reverse, 5'GCTGCGCTGATAGACATCCA3'.

The protocol was as follows: For primary amplification $\left(15\right.$ cycles of $10 \mathrm{sec}$ at $95^{\circ} \mathrm{C} ; 20 \mathrm{sec}$ at $60^{\circ} \mathrm{C}$ and $20 \mathrm{sec}$ at $72^{\circ} \mathrm{C}$ ), $5 \mu \mathrm{l}$ of genomic DNA representing each test-sample or $5 \mu \mathrm{l}$ of water (negative control) were dispensed into $0.2 \mathrm{ml}$ PCR strips and placed into a 24-well thermocycling block within the Gene-Plex robotic platform (Applied Biosystems ${ }^{\mathrm{TM}}$; Thermo Fisher Scientific, Inc.). Following the dispensing of each sample and the initiation of the assay, the following set-up process and analysis were executed by the program Assay Setup (cat. no. 9001550; Qiagen, Inc.), with the secondary amplification in PCR and the melting curve analysis being semi-automated.

Hemodynamic measurements. After 21 days following PAH induction the mice were anesthetized. Right ventricular (RV) pressures were measured using a high-fidelity pressure sensor catheter inserted directly into the right ventricle. Pressure waveforms were recorded for 2 min for each mouse using the PowerLab Chart 5 version 5.3 data acquisition system and analysis software. RV systolic pressures (RVSPs) were calculated by averaging $\geq 20$ cardiac cycles for each mouse. The RV weight-to left ventricular (LV) plus septum (S) weight ratio $(\mathrm{RV} / \mathrm{LV}+\mathrm{S})$ was calculated as the right ventricle hypertrophy index (RVHI).

Histology. The histology of PAH mice was detected using hematoxylin and eosin (H\&E) staining as previously described $(16,17)$. Briefly, the pulmonary artery tissues were perfused, inflated, separated and fixed with $4 \%$ formaldehyde at room temperature for $24 \mathrm{~h}$, embedded in paraffin, hydrated and sectioned (thickness, $5 \mu \mathrm{m}$ ) using a Leica RM2255 rotary paraffin section machine (Leica Microsystems GmbH, Wetzlar, Germany) at room temperature with a humidity of $70 \%$. Tissue sections were then dried at $60^{\circ} \mathrm{C}$, deparaffinizated by xylene and rehydrated by gradient of ethanol $(100,95,85$ and $70 \%)$ at room temperature for $5 \mathrm{~min}$. Hematoxylin (Sigma-Aldrich; Merck KGaA) staining (100 $\mu \mathrm{l})$ was performed for $5 \mathrm{~min}$ at room temperature after the section was rinsed using distilled water. The section was then rinsed with PBS. Eosin (Merck KGaA) staining was performed for 3-5 min at room temperature and then sections were rinsed using distilled water. Gradient alcohol (70, 85, 95 and 100\%) was adopted for dehydration for $5 \mathrm{~min}$ at each gradient. H\&E-staining was quantitatively evaluated by a point-counting technique in 10 randomly selected microscopic fields, at a final magnification of x200 under a 100-point grid by light microscopy. Blind analysis was performed on all sections by the same observer.

Western blot analysis. Pulmonary tissue was homogenized, lysed in a radioimmunoprecipitation buffer (Amyjet Scientific, Wuhan, China) and centrifuged to obtain the supernatant. Proteins were separated using 10\% SDS-PAGE. Total protein (20 $\mu \mathrm{g} / \mathrm{lane}$ ) was then separated on $10 \%$ SDS polyacrylamide gel and blotted onto HyBond $\mathrm{N}$ membranes (EMD Millipore, Billerica, MA, USA). Blocking was then performed with 5\% skimmed milk solution in TBS with $0.1 \%$ Tween-20 for $2 \mathrm{~h}$ at room temperature. The membranes were incubated with primary antibodies at $4^{\circ} \mathrm{C}$ overnight against PDK1, phosphorylated (p)-Akt ${ }^{\mathrm{T} 308}$, Akt, ribosomal protein S6 kinase (p70S6K; cat. no. ab8811; 1:1,000), p-p70S6K, (cat. no. ab8892; 1:1,000), proline-rich Akt1 substrate 1 (PRAS40; cat. no. ab3323; 1:1,000), p-PRAS40 (cat. no. ab5505; 1:1,000), p-S6KT229 (cat. no. ab1223; 1:1,000), p-S6T40/244 (cat. no. ab4435; 1:1,000) and S6 (cat. no. ab2334; 1:1,000) (all from Abcam, Cambridge, UK). All antibodies were purchased from Cell Signaling Technologies Inc. (Beverly, MA, USA) $\beta$-actin (cat. no. ab8227; 1:1,000; Abcam) was used as the internal reference protein. Membranes were then incubated with horseradish peroxidase-conjugated goat anti-mouse immunoglobulin G secondary antibodies (cat. no. ab6789; 1:5,000; 
A

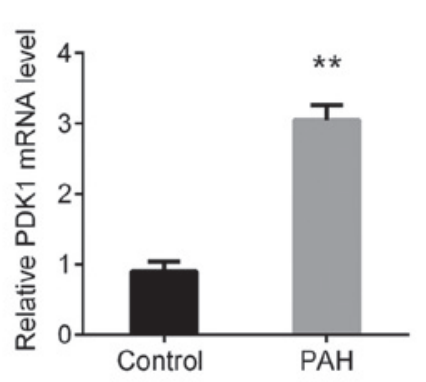

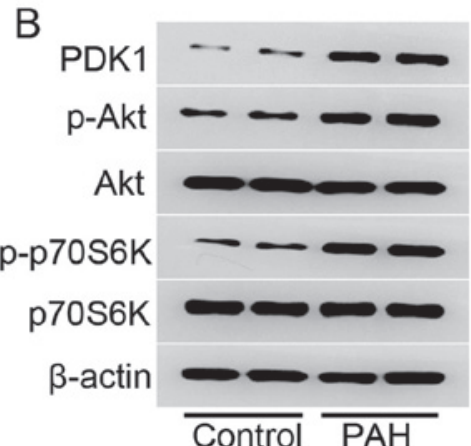

Figure 1. Expression of PDK1 in a mouse model of hypoxia-induced PAH. (A) mRNA expression of PDK1 as determined by reverse transcription-quantitative polymerase chain reaction. (B) Protein expression of PDK1, Akt, p-Akt, p70S6K and p-p70S6K in a mouse model of PAH as determined by western blot analysis. ${ }^{* *} \mathrm{P}<0.01$ vs. the control. PAH, pulmonary arterial hypertension; PDK1, phosphoinositide-dependent protein kinase-1; PAH, pulmonary arterial hypertension; Akt, protein kinase B; p-, phosphorylated; p70S6K, ribosomal protein S6 kinase.

Abcam) for $2 \mathrm{~h}$ at room temperature. The antigen-antibody complexes were visualized using an enhanced chemiluminescence detection plus kit (cat. no. PE0010; EMD Millipore) according to the manufacturer's protocol.

$R T$ - $q P C R$. Total RNA was extracted from mice lung vessels using TRIzol ${ }^{\circledR}$ (Invitrogen; Thermo Fisher Scientific, Inc., Waltham, MA, USA) and first-strand cDNA was synthesized at $37^{\circ} \mathrm{C}$ for $15 \mathrm{~min}$ (cat. no. 00345; Qiagen, Inc.). The mRNA expression of PDK1 was determined using a SYBR Premix Ex Taq $^{\text {TM }}$ kit (Takara Bio, Inc., Otsu, Japan) on an ABI 7500 fast real time PCR platform (Applied Biosystems; Thermo Fisher Scientific, Inc.). The following thermocycling conditions were used: $95^{\circ} \mathrm{C}$ for $3 \mathrm{~min}, 40$ cycles of $95^{\circ} \mathrm{C}$ for $30 \mathrm{sec}, 60^{\circ} \mathrm{C}$ for $30 \mathrm{sec}$ and $72^{\circ} \mathrm{C} 10 \mathrm{sec}$, followed by $65^{\circ} \mathrm{C}$ for $3 \mathrm{~min}$. The relative expression level was calculated using the $2^{-\Delta \Delta \mathrm{Cq}}$ method (18). All reactions were run in triplicate. $\beta$-actin was used as the internal reference gene for mRNA detection. The forward and reverse primers were as following: PDK1, forward, CTGTGA TACGGATCAGAAACCG and reverse, TCCACCAAACAA TAAAGAGTGCT; $\beta$-actin forward, GGTGTGGCATCAGGA TTCAAG and reverse, TTTCATACCGATTGCTGTTGGA.

Statistical analysis. Statistical analyses were performed using SPSS 17.0 software (SPSS, Inc., Chicago, IL, USA). All data are expressed as the mean + standard deviation from three independent experiments with each measured in triplicate. Differences between two groups and among three groups were assessed by Student's t-test and one-way analysis of variance, respectively. A post-hoc test (Tukey's test) was performed following ANOVA. $\mathrm{P}<0.05$ was considered to indicate a statistically significant difference.

\section{Results}

PDK1 is upregulated in lung tissue in a mouse model of $P A H$. PDK1 mRNA was significantly upregulated in a mouse model of hypoxia-induced PAH compared with the control as detected by RT-qPCR (Fig. 1A). The protein expression of PDK1 was increased in the PAH group compared with the control as determined by western blot analysis (Fig. 1B). It was also observed that p-Akt and p-p70S6K were increased markedly in the PAH group compared with the control, which
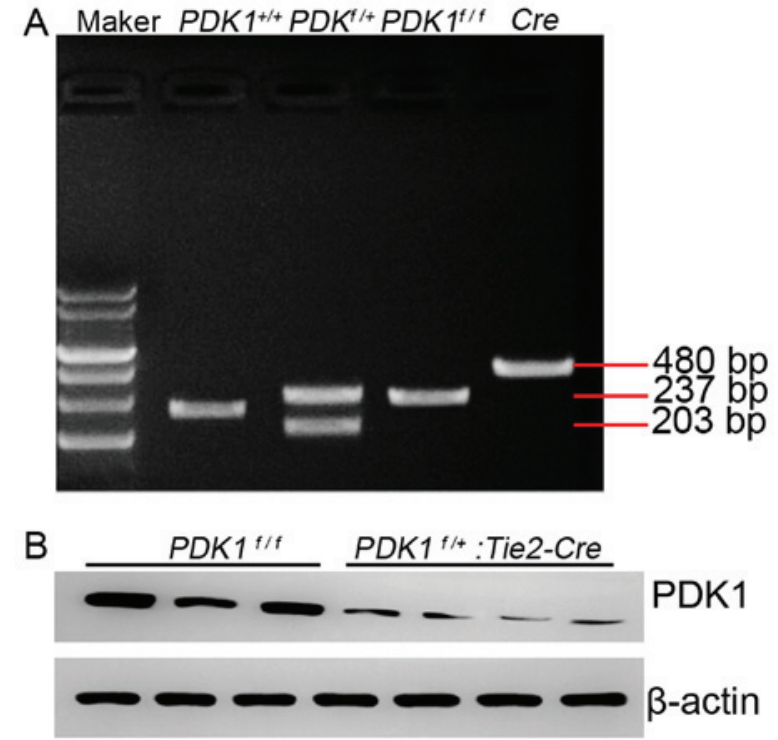

Figure 2. Identification of PDK1 genotype in mice. (A) Semi-quantitative polymerase chain reaction analysis was performed to determine the PDK1 genotypes of the mice using the tails obtained 7 days after birth. (B) Protein expression of PDK1 in the yolk sac of 5 normal type and PDK $1^{f /+}$ : Tie2-Cre mice. PDK1, phosphoinositide-dependent protein kinase-1.

indicated that the PDK1/Akt/p70S6K signaling pathway was activated.

Generation of $P D K 1^{f /+}$ genotype mice. $P D K 1^{\text {flox/flox }}$ male mice and Tie2-Cre female mice were hybridized and the tails were obtained 7 days after birth. sqPCR was performed and the embryos with different PDK1 genotypes were revealed (Fig. 2A). Mice with the $P D K l^{f /+}$ genotype had clear bi-stripe features (Fig. 2A). The protein expression of PDK1 in PDK1 knockout mice was notably reduced compared with the wild type mice (Fig. 2B), which indicated that the $P D K I^{f /+}$ : Tie2-Cre mice were successfully established.

$P D K 1^{f /+}$ genotype reduces hypoxia-induced $P A H$ in mice. After 21 days following PAH induction RVSP and RVHI $(\mathrm{RV} / \mathrm{LV}+\mathrm{S})$ were significantly increased in the experimental group compared with the control (Fig. 3A). Hypoxia induced a notable increase in the thickness of the lung vessels and the 
A

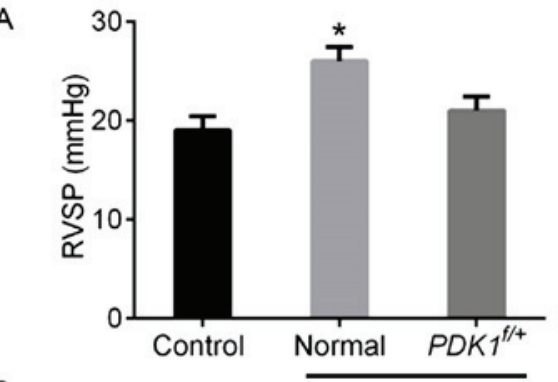

B

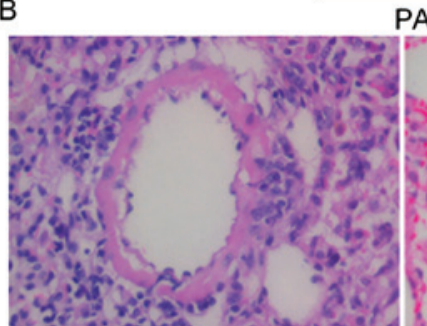

Control

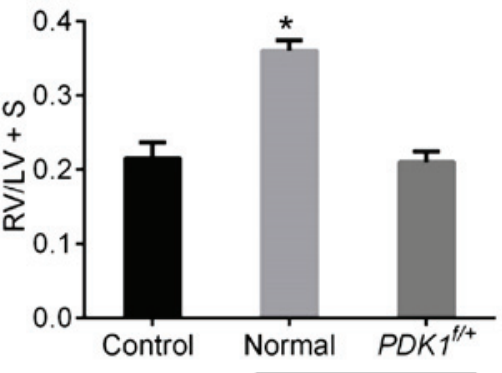

$\mathrm{PAH}$

Figure 3. PDK1 knockout limits pulmonary vascular remodeling in mice under hypoxia. (A) Hemodynamic detection of RVSP and RVHI. * $<0.05$ vs. the control. (B) Histology of pulmonary vessels. Magnification, $\mathrm{x} 40$. RVSP, right ventricular systolic pressure; RV/LV+S, right ventricular weight-to-left ventricular plus septum weight ratio; PDK1, phosphoinositide-dependent protein kinase-1. Hypoxia induced a notable increase in the thickness of the lung vessels and the proportion of muscularized pulmonary vessels. The black arrows in (B) demonstrate these changes.

proportion of muscularized pulmonary vessels (as indicated by black arrows in Fig. 3B). However, the partial deletion of PDK1 (PDK1 ${ }^{\mathrm{f} /+}$ : Tie2-Cre mice) reduced hypoxia-induced damage to the pulmonary vessels, resulting in notably lower RVSP and RVHI values compared with the control mice. The histology of the PDK $1^{f /+}$ mice was similar to that of the control group. These results demonstrated that the $P D K 1^{f /+}$ genotype may limit the procession of hypoxia-induced $\mathrm{PAH}$ in mice, potentially by reducing pulmonary vascular remodeling.

$P D K I^{f /+}$ genotype reduces hypoxia-induced activation of the PDK1/Akt/p70S6K signaling pathway. The phosphorylation levels of $\mathrm{Akt}^{\mathrm{T} 308}$, PRAS40, S6K ${ }^{\mathrm{T} 229}$ and S6 ${ }^{\mathrm{T} 240 / 244}$ were upregulated in the pulmonary vessels of the PDK $1^{f /+} \mathrm{PAH}$ group (Fig. 4). This suggested that the PDK1/Akt/p70S6K signaling pathway was activated. By contrast, the phosphorylation of these proteins in the $P D K 1^{f /+}$ group was like the control group and notably reduced compared with the normal PAH group. This indicated that the partial knockout of $P D K 1$ protected against the hypoxia-induced activation of the PDK1/Akt/p70S6K signaling pathway in mice.

\section{Discussion}

PDK1 serves an important role in activating the AGC subfamily of protein kinases, including Akt, which is essential to PAH as well as pulmonary and liver fibrosis (18-20). The results of the present study indicated that PDK1/Akt signaling promoted PAH and the partial knockout of PDK1 reduced hypoxia-induced Akt activation and $\mathrm{PAH}$ procession.

$\mathrm{PAH}$ is characterized by excessive proliferation of pVSMC and pVEC, which results in pulmonary pressure (21). The activation of Akt signaling promotes cell proliferation and survival, which accelerates disease procession and the metastasis of various types of tumor (21-24). Allard et al (24) demonstrated that the activation of Akt promoted their survival of VSMCs

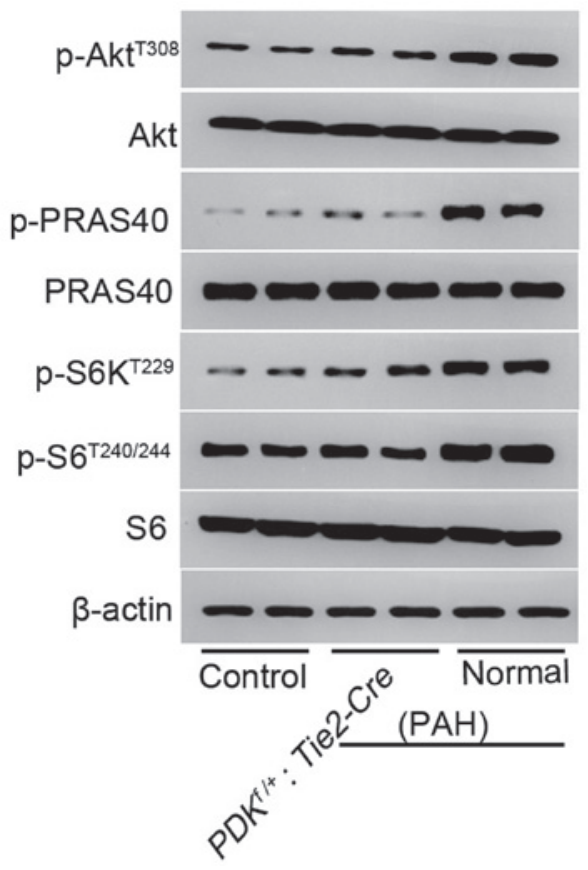

Figure 4. PDK1 knockout in mice reduces hypoxia-induced activation of the PDK1/Akt/p70S6K signaling pathway. Proteins were isolated from the mice pulmonary vessels and detected using western blot analysis. It was revealed that the partial knockout of PDK1 reduced the activation of the PDK1/Akt/p70S6K signaling pathway in hypoxia-induced PAH mice. Akt, protein kinase B; PDK1, phosphoinositide-dependent protein kinase-1; p70S6K, ribosomal protein S6 kinase; PAH, pulmonary arterial hypertension; PRAS40, proline-rich Akt1 substrate 1; p-, phosphorylated.

and inhibited the formation of intimal plaques in atherosclerosis. Tang et al (21) recently suggested that the activation of the Akt/mTOR signaling pathway and the knockout of Akt significantly reduced hypoxia-induced PAH and vascular remodeling in a mouse model. The present study demonstrated 
that hypoxia significantly upregulated the expression of PDK1 and notably upregulated the phosphorylation of Akt, whereas knockout of PDK1 notably reduced Akt phosphorylation and hypoxia-induced PAH. These results indicated that PDK1 is essential for hypoxia-induced $\mathrm{PAH}$, which is associated with the activation of Akt signals.

PDK1 phosphorylates $\mathrm{Akt}^{\mathrm{T} 308}$, which subsequently activates Akt signaling pathways, including the Akt survival pathway (25). PRAS40 (40 kDa) is a novel mTOR binding partner, which serves essential roles in the transmission of Akt signaling by mediating Akt signals to the mTORC1 kinase domain, thus inhibiting mTOR signalling (26). The Akt/mTOR signaling pathway serves important roles in extracellular remodelling (19) as well as contributing to fibrosis in non-alcoholic steatohepatitis (27) and cancer cell growth and proliferation $(28,29)$ by crosstalk with signals, including p38/mitogen activated protein kinase, 40S ribosomal protein S6 and extracellular signal-regulated kinase (30-32). Activation of mTOR signaling may be induced by hypoxia in vascular smooth muscle and endothelial cells. In addition, silencing of PRAS40 inactivated Akt and uncouples the Akt/mTOR signaling pathway. Humar et al revealed that the activation of mTOR was essential for hypoxia-mediated VSMC proliferation and angiogenesis. However, Tang et al demonstrated that mTOR was not essential for Akt activation-mediated regulation of hypoxia-induced PAH. These contradicting results mean that any association between the $\mathrm{mTOR} / \mathrm{p} 70 \mathrm{~S} 6 \mathrm{~K}$ signaling pathway and $\mathrm{PAH}$ remains unclear. The present study demonstrated that the phosphorylation of Akt, PRAS40 and p70S6K was increased in hypoxia-induced PAH mice. However, the partial knockout of PDK1 in $P D K l^{f /+}$ mice reduced the phosphorylation of these proteins and $\mathrm{PAH}$ procession, which indicated that the PDK1/Akt/PRAS40/mTOR/p70S6K signaling pathway contributed to hypoxia-induced PAH in a mouse model.

In conclusion, the present study determined that PDK1 was essential for hypoxia-induced $\mathrm{PAH}$, which was mediated by the PDK1/Akt/PRAS40/mTOR/p70S6K signaling pathway. However, it remains unclear whether the interaction or activation of the mTOR mediated signaling pathway was necessary for transmitting PDK1-mediated PAH in a mouse model. However, to some extent, this study provides useful strategies in the foreseeable future and sheds some lights in clinical diagnosis and treatment of PAH.

\section{Acknowledgements}

Not applicable.

\section{Funding}

The study was funded by the Shanghai Science and Technology Commission (grant no. 14ZR1432800) and the Scientific Research Project of Minhang District Health and Family Planning Commission of Shanghai (grant no. 2012MW02).

\section{Availability of data and materials}

The datasets used and/or analyzed during the current study are available from the corresponding author on reasonable request.

\section{Authors' contributions}

RD wrote the manuscript. ZY and YX for the discussion of and PX for discussion and comments on an earlier version of the manuscript. RD designed the experiments. ZY and YX performed the experiments and acquired the data. PX analyzed the data and prepared the manuscript. All authors read and approved the final manuscript.

\section{Ethics approval and consent to participate}

The present study was approved by the Research Ethics Committee of Shanghai Fifth People's Hospital.

\section{Patient consent for publication}

Not applicable.

\section{Competing interests}

The authors declare that they have no competing interests.

\section{References}

1. Sankhe S, Manousakidi S, Antigny F, Arthur Ataam J, Bentebbal S, Ruchon Y, Lecerf F, Sabourin J, Price L, Fadel E, et al: T-type $\mathrm{Ca}^{2+}$ channels elicit pro-proliferative and anti-apoptotic responses through impaired PP2A/Akt1 signaling in PASMCs from patients with pulmonary arterial hypertension. Biochim Biophys Acta 1864: 1631-1641, 2017.

2. Humar R, Kiefer FN, Berns H, Resink TJ and Battegay EJ: Hypoxia enhances vascular cell proliferation and angiogenesis in vitro via rapamycin (mTOR)-dependent signaling. FASEB J 16: 771-780, 2002.

3. Xia H, Diebold D, Nho R, Perlman D, Kleidon J, Kahm J, Avdulov S, Peterson M, Nerva J, Bitterman P and Henke C: Pathological integrin signaling enhances proliferation of primary lung fibroblasts from patients with idiopathic pulmonary fibrosis. J Exp Med 205: 1659-1672, 2015.

4. Lu Y, Azad N, Wang L, Iyer AK, Castranova V, Jiang BH and Rojanasakul Y: Phosphatidylinositol-3-kinase/akt regulates bleomycin-induced fibroblast proliferation and collagen production. Am J Respir Cell Mol Biol 42: 432-441, 2010.

5. Sato S, Fujita $N$ and Tsuruo T: Interference with PDK1-Akt survival signaling pathway by UCN-01 (7-hydroxystaurosporine). Oncogene 21: 1727-1738, 2002.

6. Orlacchio A, Arciuch VA and Cristofano AD: PDK1-dependent activation of AGC kinases is an absolute requirement for AKT oncogenic activity. Cancer Res 74 (Suppl 19): 4776-4776, 2014.

7. Dupuy F, Tabariès S, Andrzejewski S, Dong Z, Blagih J, Annis MG, Omeroglu A, Gao D, Leung S, Amir E, et al: PDK1-dependent metabolic reprogramming dictates metastatic potential in breast cancer. Cell Metab 22: 577-589, 2015.

8. Li W, Qian L, Lin J, Huang G, Hao N, Wei X, Wang W and Liang J: CD44 regulates prostate cancer proliferation, invasion and migration via PDK1 and PFKFB4. Oncotarget 8: 65143-65151, 2017.

9. Fang B, Zhu J, Wang Y, Geng F and Li G: MiR-454 inhibited cell proliferation of human glioblastoma cells by suppressing PDK1 expression. Biomed Pharmacother 75: 148-152, 2015.

10. Liu Y, Yang K, Sun X, Fang P, Shi H, Xu J, Xie M and Li M: MiR-138 suppresses airway smooth muscle cell proliferation through the PI3K/AKT signaling pathway by targeting PDK1. Exp Lung Res 41: 363-369, 2015.

11. Yu ZL, Wang JN, Wu XH, Xie HJ, Han Y, Guan YT, Qin Y and Jiang JM: Tanshinone IIA prevents rat basilar artery smooth muscle cells proliferation by inactivation of PDK1 during the development of hypertension. J Cardiovasc Pharmacol Ther 20: 563-571, 2015.

12. Mora A, Davies AM, Bertrand L, Sharif I, Budas GR, Jovanović S, Mouton V, Kahn CR, Lucocq JM, Gray GA, et al: Deficiency of PDK1 in cardiac muscle results in heart failure and increased sensitivity to hypoxia. EMBO J 22: 4666-4676, 2003. 
13. Belgardt BF, Husch A, Rother E, Ernst MB, Wunderlich FT, Hampel B, Klöckener T, Alessi D, Kloppenburg P and Brüning JC: PDK1 deficiency in POMC-expressing cells reveals FOXO1-dependent and-independent pathways in control of energy homeostasis and stress response. Cell Metab 7: 291-301, 2008

14. Papandreou I, Cairns RA, Fontana L, Lim AL and Denko NC: HIF-1 mediates adaptation to hypoxia by actively downregulating mitochondrial oxygen consumption. Cell Metab 3: 187-197, 2006.

15. Ciuclan L, Bonneau O, Hussey M, Duggan N, Holmes AM, Good R, Stringer R, Jones P, Morrell NW, Jarai G, et al: A novel murine model of severe pulmonary arterial hypertension. Am J Respir Crit Care Med 184: 1171-1182, 2011.

16. Song Y, Coleman L, Shi J, Beppu H, Sato K, Walsh K, Loscalzo J and Zhang YY: Inflammation, endothelial injury, and persistent pulmonary hypertension in heterozygous BMPR2-mutant mice. Am J Physiol Heart Circ Physiol 295: H677-H690, 2008.

17. Song Y, Jones JE, Beppu H, Keaney JF Jr, Loscalzo J and Zhang YY: Increased susceptibility to pulmonary hypertension in heterozygous BMPR2-mutant mice. Circulation 112: 553-562, 2005.

18. Wang J, Chu ES, Chen HY, Man K, Go MY, Huang XR, Lan HY, Sung JJ and Yu J: microRNA-29b prevents liver fibrosis by attenuating hepatic stellate cell activation and inducing apoptosis through targeting PI3K/AKT pathway. Oncotarget 6: 7325-7338, 2015

19. Abdalla M, Sabbineni H, Prakash R, Ergul A, Fagan SC and Somanath PR: The Akt inhibitor, triciribine, ameliorates chronic hypoxia-induced vascular pruning and TGF $\beta$-induced pulmonary fibrosis. Br J Pharmacol 172: 4173-4188, 2015.

20. Larson-Casey JL, Murthy S, Ryan AJ and Carter AB: Modulation of the mevalonate pathway by akt regulates macrophage survival and development of pulmonary fibrosis. J Biol Chem 289: 36204-36219, 2014.

21. Tang H, Chen J, Drennan AR, Fraidenburg DR, Song S, Sysol JR, Smith KA, Machado RF, Makino A and Yuan JX: Akt/mTOR signaling contributes to the development of pulmonary arterial hypertension, in D27. Preclinical models of pulmonary hypertension: Novel targets and pathways. Am Thoracic Soc 189: A5559, 2014.

22. Dai W, Xu X, Li S, Ma J, Shi Q, Guo S, Liu L, Guo W, Xu P, $\mathrm{He} \mathrm{Y}$, et al: SOX4 promotes proliferative signals by regulating glycolysis through AKT activation in melanoma cells. J Invest Dermatol 137: 2407-2416, 2017.
23. Dong Y, Liang G, Yuan B, Yang C, Gao R and Zhou X: MALAT1 promotes the proliferation and metastasis of osteosarcoma cells by activating the PI3K/Akt pathway. Tumor Biol 36: 1477-1486, 2015.

24. Allard D, Figg N, Bennett MR and Littlewood TD: Akt regulates the survival of vascular smooth muscle cells via inhibition of FoxO3a and GSK3. J Biol Chem 283: 19739-19747, 2008.

25. Sato S, Fujita N and Tsuruo T: Interference with PDK1-Akt survival signaling pathway by UCN-01 (7-hydroxystaurosporine). Oncogene 21: 1727-1738, 2002.

26. Vander Haar E, Lee SI, Bandhakavi S, Griffin TJ and Kim DH: Insulin signalling to mTOR mediated by the Akt/PKB substrate PRAS40. Nat Cell Biol 9: 316-323, 2007.

27. Cai CX, Buddha H, Castelino-Prabhu S, Zhang Z, Britton RS, Bacon BR and Neuschwander-Tetri BA: Activation of insulin-PI3K/Akt-p70S6K pathway in hepatic stellate cells contributes to fibrosis in nonalcoholic steatohepatitis. Dig Dis Sci 62: 968-978, 2017.

28. Seufferlein T and Rozengurt E: Rapamycin inhibits constitutive p70s6k phosphorylation, cell proliferation, and colony formation in small cell lung cancer cells. Cancer Res 56: 3895-3897, 2004.

29. Syed DN, Chamcheu JC, Khan MI, Sechi M, Lall RK, Adhami VM and Mukhtar H: Fisetin inhibits human melanoma cell growth through direct binding to p70S6K and mTOR: Findings from 3-D melanoma skin equivalents and computational modeling. Biochem Pharmacol 89: 349-360, 2014.

30. Chai X, Chu H, Yang X, Meng Y, Shi P and Gou S: Metformin increases sensitivity of pancreatic cancer cells to gemcitabine by reducing $\mathrm{CD}_{133^{+}}$cell populations and suppressing ERK/P70S6K signaling. Scientific Reports 5: 14404, 2015.

31. Li G, Shan C, Liu L, Zhou T, Zhou J, Hu X, Chen Y, Cui H and Gao N: Tanshinone IIA inhibits HIF-1 $\alpha$ and VEGF expression in breast cancer cells via mTOR/p70S6K/RPS6/4E-BP1 signaling pathway. PLoS One 10: e0117440, 2015.

32. Conejo R, de Alvaro C, Benito M, Cuadrado A and Lorenzo M: Insulinrestoresdifferentiation of Ras-transformedC2C12 myoblasts by inducing NF-kappaB through an AKT/P70S6K/p38-MAPK pathway. Oncogene 21: 3739-3753, 2002. 